\title{
Muscle Strength, Sarcopenia and Frailty Associations with Balance and Gait Parameters: A Cross-sectional Study
}

\author{
(1) Mateu Serra-Prat, (1) Elisabet Palomera \\ Consorci Sanitari del Maresme Research Unit, Mataró Hospital, Clinic of Geriatrics, Mataró, Spain
}

\begin{abstract}
Objective: While sarcopenia and frailty may lead to gait and balance impairments, their impact on these parameters is not well understood. To assess the relationship of some gait and balance parameters with sarcopenia and frailty in community-dwelling elderly people.

Materials and Methods: Observational cross-sectional study of community-dwelling people $\geq 70$ years old. Frailty and sarcopenia were defined according to Fried criteria and the criteria of the European Working Group on Sarcopenia in Older People, respectively. Balance and gait assessments included the single-leg stand test, static posturography using a force platform and static and dynamic baropodometric studies of pressures from the soles of the feet.

Results: Sixty-two patients (mean age=78 years; 50\% women) were recruited, 21\% and 14\% of whom were frail and with sarcopenia, respectively. Poor muscle strength, sarcopenia and frailty were associated with incapacity to stand on one foot for 5 seconds but not with instrumental balance assessments. Poor muscle strength and frailty were associated with longer support time, longer double support time, shorter gait and slower gait but were not associated with gait cadence. After adjusting for age and other possible confounders, the effect of frailty, but not the effect of poor muscle strength, on gait parameters disappeared. Frailty and falls were associated with a crude odds ratio of 3.4 ( $p=0.083$ ).
\end{abstract}

Conclusion: Poor muscle strength and frailty are associated with incipient balance disorders (indicating an increased risk of falls), and with gait adaptations.

Keywords: Sarcopenia, frailty, muscle strength, balance, gait

\section{Introduction}

Walking is a typical aspect of daily living and probably the human activity with the highest impact on individual functional autonomy. This complex motor behaviour involves approximately 1000 muscles, 200 bones, 100 joints and the interaction of the motor, sensory and cognitive systems (1). In aged populations, walking performance decreases mainly due to muscle strength loss and adaptations such as shorter gait and greater gait width, which may define a senile walking pattern (2). The prevalence of gait alterations increases with age; while $15 \%$ of people aged 60 years present abnormal walking characteristics, this percentage increases to $80 \%$ in people aged 85 years and older (3). The postural system integrates afferent information from the ears, eyes and joints in the central nervous system (CNS) to elaborate a motor response that maintains body balance and avoids both standing and walking falls. Changes in the CNS, vestibular, visual and attention dysfunctions and loss of muscle strength with age have all been associated with balance impairments in aged populations $(2,4,5)$. These gait and balance impairments may have severe consequences, including falls, fractures, immobility, disability or institutionalization, and may also indicate underlying diseases (6).

Loss of muscle mass and strength are closely associated with exhaustion, poor physical activity and slower gait speed, all of which are components of the frailty syndrome (7). Frailty has been defined as a state of increased vulnerability to stressors due to age-related declines in physiological reserves across neuromuscular, metabolic and immune systems (8). Balance and gait impairments may be an expression of frailty given the characteristic muscular weakness associated with this geriatric

Address for Correspondence: Mateu Serra-Prat, Consorci Sanitari del Maresme Research Unit, Mataró Hospital, Clinic of Geriatrics, Mataró, Spain Phone: +34 937417730 E-mail: mserra@csdm.cat ORCID: orcid.org/0000-0002-6554-9913

Received: Jul 16, 2019 Accepted: 0ct 02, 2019

Cite this article as: Serra-Prat M, Palomera E. Muscle Strength, Sarcopenia and Frailty Associations with Balance and Gait Parameters: A Cross-sectional Study. Eur J Geriatr Gerontol 2019;1(2):61-66

๑Copyright 2019 by the Academic Geriatrics Society / European Journal of Geriatrics and Gerontology published by Galenos Publishing House. 
syndrome. Sarcopenia and frailty are suspected to play a role in the development of gait and balance impairments, but their impact on specific parameters is not well understood.

The aim of this study was to assess the relationship between certain gait/balance parameters and sarcopenia and frailty in community-dwelling elderly patients.

\section{Materials and Methods}

\section{Design and Population}

An observational cross-sectional study was performed. Study population included community-dwelling patients aged 70 years and older. Participants that fulfilled all selection criteria and gave their informed consent in writing were recruited from outpatients visiting the Hospital of Mataró (Barcelona, Spain) from January 2013 to January 2014. Candidates were excluded if they had anyone of the following clinical conditions: life expectancy less than 3 months, dementia, severe psychiatric disorders or an inability to stand and walk. This was considered a pilot study that sought to recruit 60 subjects. The study protocol was approved by the Local Ethics Committee (exp. CEIC CSdM 49/13).

\section{Sarcopenia and Frailty Definitions}

Patients were classified as frail if they met three or more of the following five criteria (7): unintentional weight loss, exhaustion, low physical activity, slow walking speed and poor grip strength. Sarcopenia was defined according to European Working Group on Sarcopenia in Older People [EWGSOP) criteria (9): a decrease in muscle mass $(<$ mean-1 standard deviation of the reference population] accompanied by a decrease in walking speed $(\leq 0.8 \mathrm{~m} / \mathrm{s})$ or in muscle grip strength (17 kg for women and $30 \mathrm{~kg}$ for men). Muscle mass was assessed by bioimpedance analysis (Bioelectrical Impedance Analyser, EFG3 Electofluidgraph, Akern SRL), which determines fat mass, lean mass and muscle mass in kilogrammes and as a percentage of total body weight. Used as a measure of muscle strength was hand grip, assessed by a handheld JAMA dynamometer. Of three measurements made for each participant the highest value was used for this study. Patients were also asked directly about the numbers of falls experienced by them in the last 3 months.

\section{Balance and Gait Assessments}

The one-legged standing (OLS) test was used to assess whether a patient was capable of standing on one foot without help for 5 seconds. Static posturography was assessed using a force platform (Accugait, AMTI), which records displacement of the centre of pressures of the human body on the $X, Y$ and $Z$ axes. Postural balance was measured, while the subject stood on the platform, as the sweep area (SA) from the centre of pressures (in $\mathrm{cm}^{2}$ ), for both eyes open and eyes closed (Romberg test). A baropodometric study assessed static and dynamic pressures of the soles of the feet (measured in $\mathrm{N} / \mathrm{cm}^{2}$ ), and gait and its different sub phases were evaluated, at speeds that were comfortable for the patients, using a baropodometric tape (Servís Medical, model WinFDM-T). The main static baropodometry measurements, expressed as percentages, were support with the left foot, support with the right foot, total support time, support by the forefoot and support by the hindfoot. The main dynamic baropodometry measurements included the following gait cycle values: support phase duration (seconds), step duration (seconds), step length (cm), cadence (steps/second) and walking speed (meters/second).

\section{Other Study Variables}

Other factors included age, sex, comorbidities (arthrosis, diabetes, ischemic heart disease, heart failure, stroke, chronic obstructive pulmonary disease -COPD-, chronic kidney failure, chronic liver disease, Parkinson disease, depression, etc.), chronic medication, functional capacity assessed using the Barthel index, timed up-and-go test, previous falls and gait speed. Information on comorbidities and medication was obtained from each patient's electronic medical record, and all other information was obtained directly from the patient by trained healthcare professionals.

\section{Statistics}

Continuous variables were described using means and standard deviations (SDs) and categorical variables were described using percentages. Comparisons between groups (frail vs non-frail, sarcopenic vs non-sarcopenic, and poor vs adequate muscle strength according to EWGSOP cut-off points) were made using the $\mathrm{X}^{2}$ test or Fisher's exact test for categorical variables and the t-test or Mann-Whitney U test for numerical variables. Linear and logistic regression analyses assessed the effect of frailty and poor muscle strength on balance and gait parameters. Multivariate linear and logistic analyses were used to adjust the effect of frailty or poor muscle strength for age and other possible confounders (related with either frailty or poor muscle strength) on the corresponding dependent variable. Statistical significance was established as $p<0.05$.

\section{Results}

Recruited were 62 patients: 31 men and 31 women, mean age 77.9 (SD 7.5) years. These were classified as frail $(n=13 ; 21.0 \%)$, pre-frail $(n=26 ; 41.9 \%)$, and robust $(n=23 ; 37.1 \%)$ and nine $(14.5 \%)$ of them met criteria for a sarcopenia diagnosis. The main characteristics of the study sample are summarized in Table 1.

Sarcopenia was associated with age, stroke, body mass index, weight loss and poor physical exercise. On comparing patients 
with and without sarcopenia, no significant differences were observed for balance parameters, except for the capacity to stand on one foot for 5 seconds ( $33.3 \%$ vs $73.6 \% ; p=0.012$ ), nor were significant differences found for gait parameters, except for higher hindfoot pressure in both the right foot (17.0 vs $\left.30.7 \mathrm{~N} / \mathrm{cm}^{2} ; p=0.010\right)$ and left foot (17.9 vs $31.1 \mathrm{~N} /$ $\left.\mathrm{cm}^{2} ; \mathrm{p}=0.015\right)$. Among sarcopenic subjects, $77 \%$ were fallers, while this percentage was $53 \%$ in non-sarcopenic ones $(p=0.275)$.

Frailty was associated with age, comorbidities such as COPD and heart failure, the use of psychiatric drugs and poor physical exercise. Table 2 compares the main balance and gait characteristics for frail and non-frail patients and for

\begin{tabular}{|c|c|}
\hline Age in years, mean (SD) & $78.1(7.4)$ \\
\hline Sex (\% women) & $50 \%$ \\
\hline \multicolumn{2}{|l|}{ Comorbidities } \\
\hline Anxiety & $24.2 \%$ \\
\hline Depression & $32.3 \%$ \\
\hline Cancer & $12.9 \%$ \\
\hline Chronic renal failure & $9.7 \%$ \\
\hline Chronic liver disease & $1.6 \%$ \\
\hline Arthrosis or rheumatism & $72.6 \%$ \\
\hline Chronic bronchitis or COPD & $17.7 \%$ \\
\hline Asthma & $3.2 \%$ \\
\hline Diabetes & $24.2 \%$ \\
\hline Hypertension & $54.8 \%$ \\
\hline Stroke & $9.7 \%$ \\
\hline Ischemic heart disease & $14.5 \%$ \\
\hline Heart failure & $24.2 \%$ \\
\hline Cardiac arrhythmias & $25.8 \%$ \\
\hline Cataracts & $53.2 \%$ \\
\hline Deafness & $29.0 \%$ \\
\hline Dizziness & $11.3 \%$ \\
\hline Fallers & $21.0 \%$ \\
\hline Falls (n) & $0.41(0.88)$ \\
\hline Outdoor walking device users & $37.1 \%$ \\
\hline Outdoor life & $91.9 \%$ \\
\hline Outdoor walking (min/day) & $34.8(29.7)$ \\
\hline Barthel index & $96.9(9.34)$ \\
\hline \multicolumn{2}{|l|}{ Body mass index } \\
\hline Men & $28.0(5.3)$ \\
\hline Women & $28.9(5.9)$ \\
\hline \multicolumn{2}{|l|}{ Hand grip (kg) } \\
\hline Men & $28.9(8.3)$ \\
\hline Women & $17.2(6.0)$ \\
\hline
\end{tabular}

patients with poor and adequate muscle strength (according to EWGSOP criteria). Regarding balance parameters, although no significant differences were observed in SA between the frailty and muscle strength groups, significant differences were observed in the capacity to stand on one foot for 5 seconds. Regarding gait parameters, frailty and poor muscle strength were both associated with longer support time on both feet, longer double support, shorter gait length and slower gait speed, but not with gait cadence. The association of frailty with gait parameters disappeared once adjustments were made for age and other possible confounders, but the association of poor muscle strength persisted after adjustments (see Table 3). Although frailty and falls were associated with both a crude and adjusted odds ratio $(\mathrm{OR})>3$, this relationship did not achieve statistical significance.

\section{Discussion}

Our findings indicate that poor muscle strength and frailty are associated with some altered balance and gait parameters, especially capacity to stand on one foot for 5 seconds, support time when walking, gait length and gait speed. However, differences in fall prevalence between frail and non-frail patients and between patients with poor and adequate muscle strength were not statistically significant.

Regarding balance, poor muscle strength, sarcopenia and frailty were all associated with incapacity to stand on one foot for 5 seconds. However, there were no significant associations between these clinical conditions and instrumental assessments of balance obtained by posturography. This apparent disagreement between clinical and instrumental assessments of balance-which has also been reported by other authors $(10,11)$-would seem to indicate that elderly patients with sarcopenia or frailty syndrome may be able to maintain balance in the usual two-foot standing position but may have difficulties to maintain balance in more complex situations-including walking-that require balancing on a single foot. The fact that frailty was strongly related to the OLS test even when adjusted for age would point to incipient balance impairment and an increased risk of falls. This adjusted relationship was not statistically significant in the case of poor muscle strength, which can be considered an incipient phase in frailty. Balance is a complex body function influenced by multiple factors (neurological, visual, vestibular and muscular), and its preservation is essential to prevent falls (2). The cross-sectional design of our study does not allow causal relationships to be established between frailty and balance impairment, both closely related and mutually influenced. Some authors suggest that the OLS test could be used as a practical marker of frailty in community-dwelling elderly people (12). We are the opinion that balance impairment is a consequence of frailty, 
but this is a hypothesis that requires support from further longitudinal and prospective research. If balance impairment is a consequence of frailty, then preventing frailty would be a useful way to avoid balance impairment, falls and their consequences.

In relation to gait parameters, sarcopenia was only associated with higher hindfoot pressure. As far as we are aware, this is the first time this association has been described and thispossibly casual-finding is not easy to interpret. However, it may point to a relative decrease in take-off force by the contralateral forefoot in patients with sarcopenia. As for poor muscle strength and frailty, in our study these were consistently associated with different temporal and spatial gait parameters. In frail patients, support time for both feet and double support time were longer and single-foot balance time was shorter. These results, which corroborate those reported by other authors (13-15), point to a compensatory mechanism aimed at ensuring walking stability and safety. Age itself is also reported to produce similar effects (16). When the effect of frailty on support time was adjusted for age, the effect was very close to statistical significance. Other authors have reported other compensatory mechanisms in spatial gait parameters in frail patients, including an increase in the support base and in gait width $(14,17)$. Our analysis of spatial gait parameters pointed to a shorter gait in frail patients and in patients with poor muscle strength. Few studies have established a direct relationship between frailty and gait length, but our finding is consistent with other reported

Table 2. Main balance and walking characteristics for frail and non-frail patients and for patients with poor and adequate muscle strength

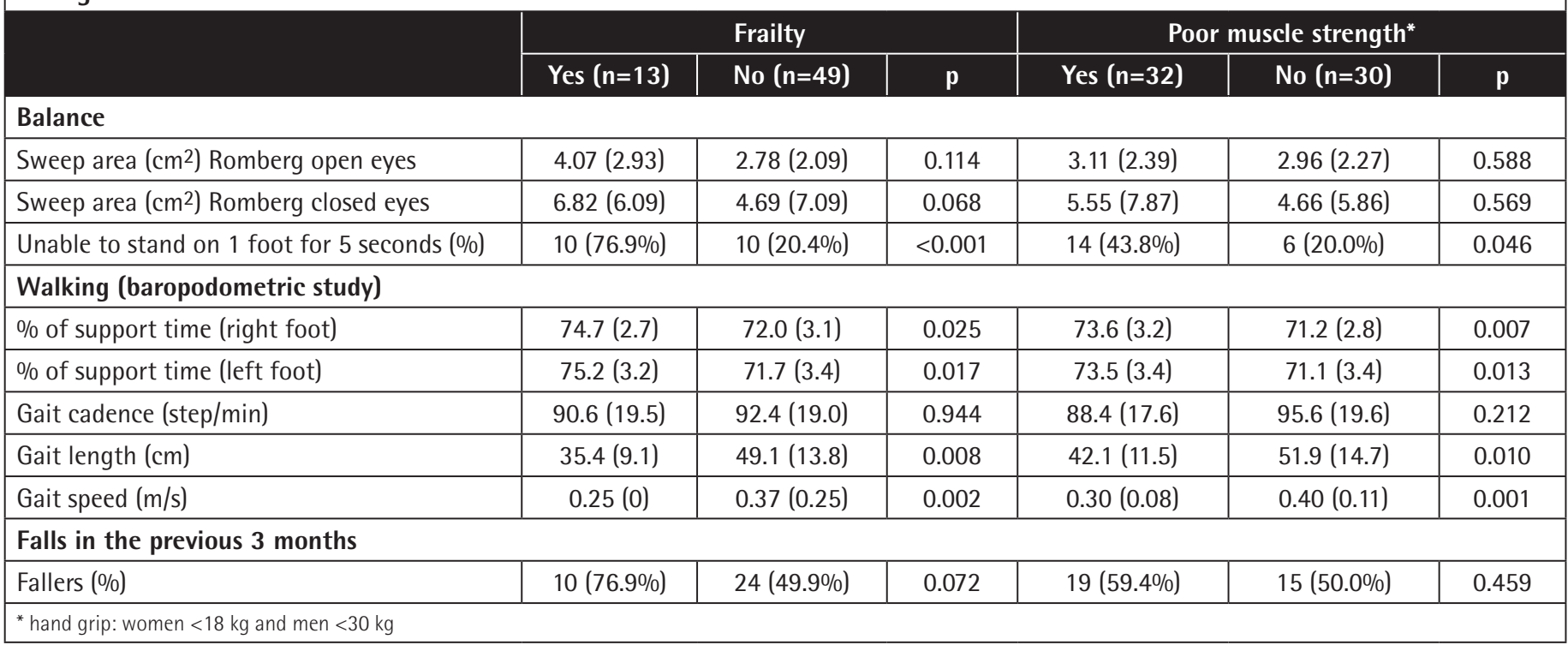

Table 3. Effect of frailty and poor muscle strength on falls, balance parameters and walking parameters (bivariate and multivariate analyses)

\begin{tabular}{|c|c|c|c|c|}
\hline & \multicolumn{2}{|c|}{ Frailty } & \multicolumn{2}{|c|}{ Poor muscle strength* } \\
\hline Linear regression analysis & Unadjusted beta (p) & Adjusted beta (p) & Unadjusted beta (p) & Adjusted beta (p) \\
\hline Sweep area $\left(\mathrm{cm}^{2}\right)$ Romberg open eyes & $1.28(0.085)$ & - & $0.156(0.794)$ & $-0.65^{d}(0.321)$ \\
\hline Sweep area $\left(\mathrm{cm}^{2}\right)$ Romberg closed eyes & $2.13(0.343)$ & $-2.11^{a}(0.349)$ & $0.881(0.623)$ & $-1.33^{a}(0.429)$ \\
\hline Gait cadence (step/min) & $-1.75(0.811)$ & $-2.30^{b}(0.772)$ & $-7.11(0.161)$ & $-7.68^{b}(0.145)$ \\
\hline Gait length $(\mathrm{cm})$ & $-13.7(0.009)$ & $-9.13^{a}(0.096)$ & $-9.75(0.008)$ & $-7.21^{\mathrm{a}}(0.051)$ \\
\hline Gait speed $(\mathrm{m} / \mathrm{s})$ & $-0.12(0.005)$ & $-0.07 a(0.100)$ & $-0.10(<0.001)$ & -0.07 a $(0.007)$ \\
\hline$\%$ of support time (right foot) & $2.75(0.024)$ & $2.31^{c}(0.059)$ & $2.41(0.004)$ & $2.13^{c}(0.012)$ \\
\hline$\%$ of support time (left foot) & $3.42(0.011)$ & $2.10^{\mathrm{a}}(0.121)$ & $2.41(0.010)$ & 1.59a $(0.081)$ \\
\hline Logistic regression analysis & Unadjusted OR (p) & Adjusted OR (p) & \multicolumn{2}{|l|}{ Unadjusted OR (p) } \\
\hline Unable to stand on 1 foot for 5 seconds & $12.5(0.001)$ & $6.41^{b}(0.022)$ & $3.12(0.050)$ & $1.84 b(0.345)$ \\
\hline Faller & $3.47(0.083)$ & $3.12^{\mathrm{a}}(0.152)$ & $1.46(0.459)$ & $1.25^{b}(0.682)$ \\
\hline $\begin{array}{l}{ }^{*} \text { hand grip: women }<18 \mathrm{~kg} \text {; men }<30 \mathrm{~kg} \text {; adju } \\
\text { physical exercise/week } \\
\text { OR: Odds ratio }\end{array}$ & se of psychiatric & & & \\
\hline
\end{tabular}


results on differences in gait length between fallers and nonfallers $(6,15)$. A systematic review, moreover, indicates that decreased gait length is a typical feature of ageing (1). Even after adjustment for age, we observed a clinically relevant and statistically significant association between frailty and gait length.

Our study also showed that frailty was associated with previous falls, for a crude $O R=3.5(p=0.083)$ and an adjusted $O R=3.1$ $(p=0.152)$. The magnitude of the association and its nearness to statistical significance would suggest that the lack of statistical significance is due to low statistical power resulting from a small sample size. Several studies have investigated frailty as a risk factor for falls, reporting diverse and not always conclusive results. A meta-analysis that pooled the results of 11 prospective studies concluded that frailty is a significant predictor of future falls among community-dwelling older people, especially men; and an observational study in 324 community-dwelling elderly people pointed to a strong relationship between muscle strength and falls in both men and women $(18,19)$. However, in our study poor muscle strength was not found to be associated with falls, whether because of a lack of statistical power or because of the strength cut-off point used.

\section{Study Limitations}

Regarding limitations of our study, the first one, as already mentioned, is the small sample size, which compromises the power to detect statistically significant relationships or differences between groups. Secondly, we cannot assume that the study sample is representative of the general population aged 70 years and older because only outpatients were recruited. Although this lack of representativeness did not allow sarcopenia or frailty prevalence to be established, it did allow a comparison of balance and gait parameters between sarcopenia and frailty groups. Thirdly, the sarcopenia and frailty groups were not homogeneous in terms of age, a factor associated with balance and gait parameters. The multivariate analysis was accordingly adjusted for age as a possible confounder. Finally, the cross-sectional design did not allow causal relationships to be established between sarcopenia and frailty on the one hand, and on the other hand, balance, gait and falls, in relation to which we suggest that prospective studies are required.

\section{Conclusion}

Poor muscle strength and frailty are associated with incipient balance disorders and with walking adaptations aimed at gaining stability that result in slower gait. While these findings would suggest that poor muscle strength and frailty are risk factors for falls in aged populations, further prospective and well-powered studies are necessary to provide more conclusive evidence.

\section{Acknowledgements}

We thank the Spanish Ministry of Health, Instituto de Salud Carlos III, Fondo de Investigación Sanitaria for funding.

\section{Ethics}

Ethics Committee Approval: The study protocol was approved by Consorci Sanitari del Maresme Research Unit, Mataró Hospital, Clinic of Geriatrics, Mataró, Spain, the Local Ethics Committee (exp. CEIC CSdM 49/13).

Informed Consent: Participants that fulfilled all selection criteria and gave their informed consent in writing were recruited from outpatients visiting the Hospital of Mataró (Barcelona, Spain) from January 2013 to January 2014.

Peer-review: Internally and externally peer-reviewed.

\section{Authorship Contributions}

Concept: M.S.P., Design: M.S.P., Data Collection or Processing: E.P., Analysis or Interpretation: E.P., Literature Search: M.S.P., Writing: M.S.P.,

Conflict of Interest: All authors declare that they have no conflict of interest in this study.

Financial Disclosure: This study was funded by grants from the Spanish Ministry of Heath, Instituto de Salud Carlos III, Fondo de Investigación Sanitaria (FIS, PI 13/00931).

\section{References}

1. Aboutorabi A, Arazpour M, Bahramizadeh M, Hutchins SW, Fadayevatan R. The effect of aging on gait parameters in able-bodied older subjects: a literature review. Aging Clin Exp Res 2016;28:393-405.

2. Snijders $A H$, van de Warrenburg BP, Giladi N, Bloem BR. Neurological gait disorders in elderly people: clinical approach and classification. Lancet Neurol 2007;6:63-74

3. Verghese J, LeValley A, Hall CB, Katz MJ, Ambrose AF, Lipton RB. Epidemiology of gait disorders in community-residing older adults. J Am Geriatr Soc 2006;54:255-261.

4. Wiesmeier IK, Dalin D, Maurer C. Elderly Use Proprioception Rather than Visual and Vestibular Cues for Postural Motor Control. Front Aging Neurosci $2015 ; 7: 97$.

5. Cofré Lizama LE, Pijnappels M, Rispens SM, Reeves NP, Verschueren SM, van Dieën JH. Mediolateral balance and gait stability in older adults. Gait Posture 2015;42:79-84.

6. Mortaza N, Abu Osman NA, Mehdikhani N. Are the spatio-temporal parameters of gait capable of distinguishing a faller from a non-faller elderly? Eur J Phys Rehabil Med 2014;50:677-691.

7. Fried LP, Tangen $C M$, Walston J, Newman AB, Hirsch C, Gottdiener J, Seeman T, Tracy R, Kop WJ, Burke G, McBurnie MA; Cardiovascular Health Study Collaborative Research Group. Frailty in older adults: evidence for a phenotype. J Gerontol A Biol Sci Med Sci 2001;56:M146-M156.

8. Morley J, Vellas B, van Kan GA, Anker SD, Bauer JM, Bernabei R, Cesari M, Chumlea WC, Doehner W, Evans J, Fried LP, Guralnik JM, Katz PR, Malmstrom TK, McCarter RJ, Robledo LMG, Rockwood K, von Haehling S Vandewoude MF, Walston J. Frailty consensus: a call to action. J Am Med DirAssoc 2013;14:392-397. 
9. Cruz-Jentoft AJ, Baeyens JP, Bauer JM, Boirie $Y$, Cederholm T, Landi $F$, Martin FC, Michel JP, Rolland Y, Schneider SM, Topinková E, Vandewoude M, Zamboni M; European Working Group on Sarcopenia in Older People. Sarcopenia: European consensus on definition and diagnosis: Report of the European Working Group on Sarcopenia in Older People. Age Ageing 2010;39:412-423.

10. Thapa PB, Gideon P, Fought RL, Kormicki M, Ray WA. Comparison of clinical and biomechanical measures of balance and mobility in elderly nursing home residents. J Am Geriatr Soc 1994;42:493-500.

11. Ringsberg K, Gerdhem P, Johansson J, Obrant KJ. Is there a relationship between balance, gait performance and muscular strength in 75-year-old women? Age Ageing 1999;28:289-293.

12. Michikawa T, Nishiwaki Y, Takebayashi T, Toyama Y. One-leg standing test for elderly populations. J Orthop Sci 2009;14:675-685.

13. Kressig RW, Gregor RJ, Oliver A, Waddell D, Smith W, O'Grady M, Curns AT, Kutner M, Wolf SL. Temporal and spatial features of gait in older adults transitioning to frailty. Gait Posture 2004;20:30-35.
14. Montero-Odasso M, Muir SW, Hall M, Doherty TJ, Kloseck M, Beauchet 0 , Speechley M. Gait variability is associated with frailty in communitydwelling older adults. J Gerontol A Biol Sci Med Sci 2011;66:568-576.

15. Schwenk M, Howe C, Saleh A, Mohler J, Grewal G, Armstrong D, Najafi B. Frailty and technology: a systematic review of gait analysis in those with frailty. Gerontology 2014;60:79-89.

16. Hollman JH, McDade EM, Petersen RC. Normative spatiotemporal gait parameters in older adults. Gait Posture 2011;34:111-118.

17. Freire Junior RC, Porto JM, Rodrigues NC, Brunelli RM, Braga LF, de Abreu DC. Spatial and temporal gait characteristics in pre-frail communitydwelling older adults. Geriatr Gerontol Int 2016;16:1102-1108.

18. Kojima G. Frailty as a Predictor of Future Falls Among Community-Dwelling Older People: A Systematic Review and Meta-Analysis. J Am Med Dir Assoc 2015; 16:1027-1033.

19. Serra-Prat M, Papiol M, Vico J, Palomera E, Bartolomé M, Burdoy E. Factors associated with poor muscle mass and strength in a community-dwelling elderly population: a cross-sectional study. J Gerontol Geriatr Res 2017;6:2. 